\title{
Dynamic behaviors for a delay Lasota-Wazewska model with feedback control on time scales
}

\author{
Xiaoying Chen ${ }^{1 *}$, Chunling Shi ${ }^{1}$ and Danhong Wang ${ }^{2}$
}

\section{"Correspondence:}

snailkitty@126.com

1 Department of Computer

Engineering, Fuzhou University

Zhicheng College, Fuzhou, P.R.

China

Full list of author information is

available at the end of the article

\section{Springer}

\begin{abstract}
In this paper, a delay Lasota-Wazewska system with feedback control on time scales is proposed. Firstly, by using some differential inequalities on time scales, sufficient conditions which ensure the permanence of the system are obtained. Secondly, by means of the fixed point theory and the exponential dichotomy of linear dynamic equations on time scales, some sufficient conditions for the existence of unique almost periodic solution are obtained. Moreover, exponential stability of the almost periodic positive solution is investigated by applying the Gronwall inequality. Finally, numeric simulations are carried out to show the feasibility of the main results.
\end{abstract}

MSC: 34C25; 92D25; 34D20; 34D40

Keywords: Lasota-Wazewska model; Time scales; Feedback control; Exponential dichotomy; Permanence

\section{Introduction}

Today the dynamical systems are classified into some types, among which discrete systems and continuous systems are two important types. But it is troublesome to study the solutions for discrete and continuous systems, respectively. Therefore, it is more significant to study a biological model which can effectively unify the discrete time and continuous time. The theory of time scales was initiated by Stefan Hilger in his Ph.D. thesis in 1988, providing a rich theory that unified and extended discrete and continuous analysis [1]. The theory of time scale calculus and dynamic equation on time scales provides us with a powerful tool for attacking such mixed processes. There has been considerable work done on the study of the almost periodicity and stability of the systems on time scales. Many interesting and important results can be found in many articles; for example, see [2-10] and the references cited therein.

In real world phenomenon, ecosystems are often disturbed by unpredictable forces which can result in changes in the biological parameters such as survival rates. The disturbance functions are called control variables in the language of control. In 1993, a feedback control variable was introduced into the Logistic models by Gopalsamy and Weng [11]. They discussed the asymptotic behavior of solution in Logistic models with feedback con-

(c) The Author(s) 2020. This article is licensed under a Creative Commons Attribution 4.0 International License, which permits use, sharing, adaptation, distribution and reproduction in any medium or format, as long as you give appropriate credit to the original author(s) and the source, provide a link to the Creative Commons licence, and indicate if changes were made. The images or other third party material in this article are included in the article's Creative Commons licence, unless indicated otherwise in a credit line to the material. If material is not included in the article's Creative Commons licence and your intended use is not permitted by statutory regulation or exceeds the permitted use, you will need to obtain permission directly from the copyright holder. To view a copy of this licence, visit http://creativecommons.org/licenses/by/4.0/. 
trols. The feedback control models play an important and fundamental role. We also refer to Fan [7], Chen [12], Li [13] and [14-22] for further study on systems with feedback control.

In 1976, Wazewska-Czyzewska, Lasota [23] investigated the Lasota-Wazewska model

$$
x^{\prime}(t)=-a x(t)+b e^{-\beta x(t-\tau)}
$$

which described the survival of red blood cells in animals. Some generalized models have been discussed by many authors; see [24-28]. Because of the various effects of the environmental factors in real life environment (e.g. food supplies, seasonal effects of weather, etc.), it is more practical and meaningful to investigate the biological model with almost periodic coefficients. Besides, permanence is affected by such factors as environment, attack rates, and so on. The permanence of ecosystems will play an important role to predict the change trend of populations in the long run. Many authors [7, 12, 21, 29, 30] have investigated the permanence of biological systems. However, to the best of the authors' knowledge, to this day, still no scholars consider the permanence of the Lasota-Wazewska model with feedback control on time scales.

Motivated by the aforementioned discussion, in the present paper, we first propose an almost periodic Lasota-Wazewska model with feedback control and multiple time-varying delays on time scales:

$$
\begin{aligned}
x^{\Delta}(t)= & -\alpha(t) x(t)+\sum_{j=1}^{m} \beta_{j}(t) e^{-\gamma_{j}(t) x\left(t-\tau_{j}(t)\right)} \\
& -c(t) u(t-\theta(t)) x(t), \quad j=1,2, \ldots, m, \\
u^{\Delta}(t)= & -a(t) u(t)+b(t) x(t-\eta(t)),
\end{aligned}
$$

where $t \in \mathbb{T}$, $\mathbb{T}$ is an almost periodic time scale, $x(t)$ denotes the number of red blood cells at time $t . \tau_{j}(t)$ is the time required to produce a red blood cell. $u(t)$ is the control variable at time $t$. About more details of the system (1.2), we can refer [23-26].

In this work, we aim to investigate the permanence of the Lasota-Wazewska timescale model with multiple time-varying delays and feedback control. Of particular interest is the question of whether or not the feedback control variables can affect the permanence of the timescale system. Furthermore, using the exponential dichotomy of linear dynamic equations on time scales and a fixed point theorem on time scales, we study the existence and uniqueness of almost periodic solutions for the model. The exponential stability of the almost periodic solution of the model is obtained by the Gronwall inequality. Our results obtained in this paper are completely new and complement the previously known result [28], which studied the almost periodic solutions for a Lasota-Wazevska model in continuous systems. The results derived in this paper are meaningful.

The paper is arranged as follows. In Sect. 2, we introduce some notations and definitions which are needed in later sections. The permanence and almost periodic solutions of system (1.2) is investigated in Sects. 3 and 4, respectively. An example together with its numeric simulations is presented in Sect. 5 to show the feasibility of the main results. We end this paper by a briefly discussion. 


\section{Preliminaries}

In this section, we shall give some notations, definitions and lemmas which are used in what follows. The reader is assumed to be familiar with some basic properties about time scales and one can find in Bohner and Peterson's book [30] most of the materials needed are available in this paper.

Let $\mathbb{T}$ be a nonempty closed subset (time scale) of $\mathbb{R}$. Since almost periodic functions defined on $\mathbb{T}$ are bounded, we use the notations

$$
h^{+}=\sup _{t \in \mathbb{T}}\{h(t)\}, \quad h^{-}=\inf _{t \in \mathbb{T}}\{h(t)\}
$$

where $h(t)$ is an almost periodic function.

Definition 2.1 ([30]) For $f: \mathbb{T} \rightarrow \mathbb{R}$, we define $f^{\Delta}(t)$ to be the number (if it exists) with the property that, for any given $\varepsilon>0$, there exists a neighborhood $U$ of $t$ such that

$$
|(f(\sigma(t))-f(s))-(\sigma(t)-s)|<\varepsilon|\sigma(t)-s|, \quad \text { for all } s \in U \text {. }
$$

We call $f^{\Delta}(t)$ the delta (or Hilger) derivative of $f$ at $t$. If $F^{\Delta}(t)=f(t)$, then we define the delta integral by $\int_{r}^{t} f(s) \Delta s=F(t)-F(r)$ for $t, r \in \mathbb{T}$.

We define the set $\mathfrak{R}^{+}=\mathfrak{R}^{+}(\mathbb{T}, \mathbb{R})=\{r \in \mathfrak{R}: 1+\mu(t) r(t)>0, \forall t \in \mathbb{T}\}$.

Lemma 2.1 ([31]) Assume that $a>0, b>0$ and $-a \in \mathfrak{R}^{+}$. Then

$$
y^{\Delta}(t) \geq(\leq) b-a y(t), \quad y(t)>0, t \in\left[t_{0}, \infty\right)_{\mathbb{T}}
$$

implying

$$
y(t) \geq(\leq) \frac{b}{a}\left[1+\left(\frac{a y\left(t_{0}\right)}{b}-1\right) e_{(-a)}\left(t, t_{0}\right)\right], \quad t \in\left[t_{0}, \infty\right)_{\mathbb{T}} .
$$

Lemma 2.2 ([31]) Assume that $a>0, b>0$. Then

$$
y^{\Delta}(t) \geq(\leq) b-a y(\sigma(t)), \quad y(t)>0, t \in\left[t_{0}, \infty\right)_{\mathbb{T}}
$$

implying

$$
y(t) \geq(\leq) \frac{b}{a}\left[1+\left(\frac{a y\left(t_{0}\right)}{b}-1\right) e_{\ominus a}\left(t, t_{0}\right)\right], \quad t \in\left[t_{0}, \infty\right)_{\mathbb{T}} .
$$

Definition 2.2 ([32]) Let $\Gamma$ be a collection of sets which is constructed by subsets of $\mathbb{R}$. A time scale $\mathbb{T}$ is called an almost periodic time scale with respect to $\Gamma$, if

$$
\Gamma^{*}=\left\{ \pm \tau \in \bigcap_{\Lambda \in \Gamma}: t \pm \tau \in \mathbb{T}, \forall t \in \mathbb{T}\right\} \neq \varnothing
$$

and $\Gamma^{*}$ is called the smallest almost periodic set of $\mathbb{T}$. Obviously, if $\mathbb{T}$ is an almost periodic time scale, then $\inf \mathbb{T}=-\infty$ and $\sup \mathbb{T}=+\infty$. 
Definition 2.3 ([32]) Let $\mathbb{T}$ is an almost periodic time scale with respect to $\Gamma$. A function $f(t) \in C\left(\mathbb{T}, \mathbb{R}^{n}\right)$ is called almost periodic if for any given $\varepsilon>0$, the set $E(f, \varepsilon)=\left\{\tau \in \Gamma^{*}\right.$ : $|f(t+\tau)-f(t)|<\varepsilon, \forall t \in \mathbb{T}\}$ is relatively dense in $\mathbb{T}$; that is, for any given $\varepsilon>0$, there exists a real number $l=l(\varepsilon)>0$ such that each interval of length $l$ contains at least one $\tau=\tau(\varepsilon) \in$ $E(f, \varepsilon)$ satisfying $|f(t+\tau)-f(t)|<\varepsilon, \forall t \in \mathbb{T}$.

The set $E(f, \varepsilon)$ is called $\varepsilon$-translation set of $f(t), \tau$ is called $\varepsilon$-translation number of $f(t)$ and $l(\varepsilon)$ is called the contain interval length of $E(f, \varepsilon)$.

Definition 2.4 ([32,33]) Let $Q(t)$ be $n \times n$ rd-continuous matrix function on $\mathbb{T}$. The linear system

$$
x^{\Delta}(t)=Q(t) x(t), \quad t \in \mathbb{T}
$$

is said to admit an exponential dichotomy on $\mathbb{T}$ if there exist positive constant $\kappa, \alpha$, projection $P$ and the fundamental solution matrix $X(t)$ of $(2.1)$ satisfying

$$
\begin{aligned}
& \left|X(t) P X^{-1}(\sigma(s))\right| \leq \kappa e_{\ominus \alpha}(t, \sigma(s)) \quad \text { for } t \geq \sigma(s), s, t \in \mathbb{T}, \\
& \left|X(t)(I-P) X^{-1}(\sigma(s))\right| \leq \kappa e_{\ominus \alpha}(\sigma(s), t) \quad \text { for } t \leq \sigma(s), s, t \in \mathbb{T},
\end{aligned}
$$

where $|\cdot|$ is a matrix norm on $\mathbb{T}$, that is, if $Q=\left(a_{i j}\right)_{n \times m}$ then we can take $|Q|=$ $\left(\sum_{i=1}^{n} \sum_{j=1}^{n}\left|a_{i j}\right|^{2}\right)^{\frac{1}{2}}$.

Consider the almost periodic system

$$
x^{\Delta}(t)=Q(t) x(t)+g(t), \quad t \in \mathbb{T},
$$

where $Q(t)$ is an almost periodic matrix function, $g(t)$ is an almost periodic vector function.

Lemma 2.3 ([5]) If the linear system (2.1) admits an exponential dichotomy, then the almost periodic system (2.2) has an almost periodic solution $x(t)$ as follows:

$$
x(t)=\int_{-\infty}^{t} X(t) P X^{-1}(\sigma(s)) g(s) \Delta s-\int_{t}^{+\infty} X(t)(I-P) X^{-1}(\sigma(s)) g(s) \Delta s, \quad t \in \mathbb{T} .
$$

\section{The permanence}

In view of the biological significance, we also assume that the coefficient functions $\alpha, \beta_{j}, \gamma_{j}, \tau_{j},(j=1,2, \ldots, m), a, b, c, \theta$ and $\eta: \mathbb{T} \rightarrow \mathbb{R}^{+}$are all bounded rd-continuous and

$$
\begin{aligned}
& \min \left\{\alpha^{-}, \beta_{j}^{-}, \gamma_{j}^{-}, \tau_{j}^{-}, a^{-}, b^{-}, c^{-}, \theta^{-}, \eta^{-}\right\}>0, \\
& \max \left\{\alpha^{+}, \beta_{j}^{+}, \gamma_{j}^{+}, \tau_{j}^{+}, a^{+}, b^{+}, c^{+}, \theta^{+}, \eta^{+}\right\}<+\infty .
\end{aligned}
$$

The initial condition of system (1.2) is of the form

$$
\begin{aligned}
& x(s)=\phi(s)>0, \quad s \in[-\tau, 0)_{\mathbb{T}}, \phi \in C_{\mathrm{rd}}\left([-\tau, 0)_{\mathbb{T}}, \mathbb{R}\right), \phi(0)>0, \\
& u(s)=\varphi(s)>0, \quad s \in[-\tau, 0)_{\mathbb{T}}, \varphi \in C_{\mathrm{rd}}\left([-\tau, 0)_{\mathbb{T}}, \mathbb{R}\right), \varphi(0)>0,
\end{aligned}
$$


where $\tau=\max \left\{\tau_{j}^{+}, \theta^{+}, \eta^{+}\right\}(j=1,2, \ldots, m),[-\tau, 0)_{\mathbb{T}}=[-\tau, 0) \cap \mathbb{T}$.

Set

$$
\begin{aligned}
& M_{1}=\frac{\sum_{j=1}^{m} \beta_{j}^{+}}{\alpha^{-}}, \quad M_{2}=\frac{b^{+} M_{1}}{a^{-}}, \\
& m_{1}=\frac{\sum_{j=1}^{m} \beta_{j}^{-} e^{-\gamma_{j}^{+} M_{1}}-c^{+} M_{1} M_{2}}{\alpha^{+}}, \quad m_{2}=\frac{b^{-} m_{1}}{a^{+}} .
\end{aligned}
$$

Theorem 3.1 Let $(x(t), u(t))^{T}$ be any positive solution of system (1.2) with initial condition (3.1). Assume that

$\left(H_{1}\right) \sum_{j=1}^{m} \beta_{j}^{-} e^{-\gamma_{j}^{+} M_{1}}>c^{+} M_{1} M_{2}$

holds, then system (1.2) is permanent, that is, any positive solution $(x(t), u(t))^{T}$ of system (1.2) satisfies

$$
\begin{aligned}
& m_{1} \leq \liminf _{t \rightarrow+\infty} x(t) \leq \limsup _{t \rightarrow+\infty} x(t) \leq M_{1} \\
& m_{2} \leq \liminf _{t \rightarrow+\infty} u(t) \leq \limsup _{n \rightarrow+\infty} u(t) \leq M_{2} .
\end{aligned}
$$

Proof Assume that $(x(t), u(t))^{T}$ is any positive solution of system (1.2) with initial condition (3.1). From the first equation of system (1.2), it immediately follows that

$$
x^{\Delta}(t) \leq-\alpha(t) x(\sigma(t))+\sum_{j=1}^{m} \beta_{j}^{+} .
$$

By Lemma 2.2, we have

$$
\limsup _{t \rightarrow+\infty} x(t) \leq \frac{\sum_{j=1}^{m} \beta_{j}^{+}}{\alpha^{-}}:=M_{1} .
$$

Let $\varepsilon>0$ be any small enough positive constant, there exists $T_{1}>0$ such that

$$
x(t)<M_{1}+\varepsilon, \quad \forall t>T_{1} .
$$

From the second equation of (1.2), we get

$$
u^{\Delta}(t)<-a^{-} u(t)+b^{+}\left(M_{1}+\varepsilon\right), \quad \forall t \in\left[T_{1}+\tau,+\infty\right)_{\mathbb{T}} .
$$

By applying Lemma 2.1, it follows that

$$
\limsup _{t \rightarrow+\infty} u(t) \leq \frac{b^{+}\left(M_{1}+\varepsilon\right)}{a^{-}} .
$$

Setting $\varepsilon \rightarrow 0$, the above inequality leads to

$$
\limsup _{t \rightarrow+\infty} u(t) \leq \frac{b^{+} M_{1}}{a^{-}}:=M_{2} .
$$


For any positive constant $\varepsilon$ small enough, there exists a $T_{2}>T_{1}+\tau$ such that

$$
u(t)<M_{2}+\varepsilon, \quad \forall t \in\left[T_{2},+\infty\right)_{\mathbb{T}} .
$$

On the other hand, from the first equation of system (1.2), we have

$$
x^{\Delta}(t)>-\alpha^{+} x(\sigma(t))+\sum_{j=1}^{m} \beta_{j}^{-} e^{-\gamma_{j}^{+}\left(M_{1}+\varepsilon\right)}-c^{+}\left(M_{1}+\varepsilon\right)\left(M_{2}+\varepsilon\right), \quad \forall t \in\left[T_{2}+\tau,+\infty\right)_{\mathbb{T}} .
$$

By Lemma 2.2, we can get

$$
\liminf _{t \rightarrow+\infty} x(t) \geq \frac{\sum_{j=1}^{m} \beta_{j}^{-} e^{-\gamma_{j}^{+}\left(M_{1}+\varepsilon\right)}-c^{+}\left(M_{1}+\varepsilon\right)\left(M_{2}+\varepsilon\right)}{\alpha^{+}}
$$

Setting $\varepsilon \rightarrow 0$, the above inequality leads to

$$
\liminf _{t \rightarrow+\infty} x(t) \geq \frac{\sum_{j=1}^{m} \beta_{j}^{-} e^{-\gamma_{j}^{+} M_{1}}-c^{+} M_{1} M_{2}}{\alpha^{+}}:=m_{1} \text {. }
$$

Then, for an arbitrarily small positive constant $\varepsilon>0$, there exists a $T_{3}>T_{2}+\tau$ such that

$$
x(t)>m_{1}-\varepsilon, \quad \forall t \in\left[T_{3},+\infty\right)_{\mathbb{T}} .
$$

From the second equation of system (1.2), it follows that

$$
u^{\Delta}(t)>-a^{+} u(t)+b^{-}\left(m_{1}-\varepsilon\right), \quad \forall t \in\left[T_{3}+\tau,+\infty\right)_{\mathbb{T}} .
$$

By Lemma 2.1, we can get

$$
\liminf _{t \rightarrow+\infty} u(t) \geq \frac{b^{-}\left(m_{1}-\varepsilon\right)}{a^{+}}
$$

Setting $\varepsilon \rightarrow 0$, the above inequality leads to

$$
\liminf _{t \rightarrow+\infty} u(t) \geq \frac{b^{-} m_{1}}{a^{+}}:=m_{2}
$$

Equations (3.2), (3.3), (3.4) and (3.5) show that if the condition $\left(H_{1}\right)$ holds, then system (1.2) is permanent.

Remark 3.1 If $\left(H_{1}\right)$ holds, system (1.2) is permanent. Theorem 3.1 revealed that the feedback control variables have no influence on the permanence of system (1.2).

\section{The almost periodic positive solution}

In this section, we state and prove our main results concerning of the existence and exponential stability of positive almost periodic solutions of (1.2).

Set $\mathbb{X}=\left\{\varphi \in C\left(\mathbb{T}, \mathbb{R}^{2}\right): \varphi=\left(\varphi_{1}, \varphi_{2}\right)\right\}$ is an almost periodic function on $\mathbb{T}$ with the norm $\|\varphi\|_{\mathbb{T}}=\sup _{t \in \mathbb{T}}\|\varphi(t)\|$, where $\|\varphi(t)\|=\max \left\{\left|\varphi_{1}(t)\right|,\left|\varphi_{2}(t)\right|\right\}$, then $\mathbb{X}$ is a Banach space. 
Theorem 4.1 Assume that $\left(H_{1}\right)$ holds. Suppose further that

$\left(H_{2}\right) \max \left\{\frac{1}{\alpha^{-}}\left(\sum_{j=1}^{m} \beta_{j}^{+} \gamma_{j}^{+}+c^{+}\left(M_{1}+M_{2}\right)\right), \frac{b^{+}}{a^{-}}\right\}<1$

holds, then system (1.2) has a positive almost periodic solution in the region $\mathbb{X}^{*}=\{\varphi=$ $\left.\left(\varphi_{1}, \varphi_{2}\right)^{T}, m_{i} \leq \varphi_{i} \leq M_{i}, t \in \mathbb{T}, i=1,2.\right\}$.

Proof For any given $\varphi \in \mathbb{X}$, the following almost periodic dynamic system is considered:

$$
\begin{aligned}
x^{\Delta}(t)= & -\alpha(t) x(t)+\sum_{j=1}^{m} \beta_{j}(t) e^{-\gamma_{j}(t) \varphi_{1}\left(t-\tau_{j}(t)\right)} \\
& -c(t) \varphi_{2}(t-\theta(t)) \varphi_{1}(t), \quad j=1,2, \ldots, m, \\
u^{\Delta}(t)= & -a(t) u(t)+b(t) \varphi_{1}(t-\eta(t)) .
\end{aligned}
$$

Because $\inf _{t \in \mathbb{T}} \alpha(t)=\alpha^{-}>0, \inf _{t \in \mathbb{T}} a(t)=a^{-}>0$, the linear system

$$
\begin{aligned}
& x^{\Delta}(t)=-\alpha(t) x(t), \\
& u^{\Delta}(t)=-a(t) u(t),
\end{aligned}
$$

admits an exponential dichotomy on $\mathbb{T}$. Thus, by Lemma 2.3 , we find that system (4.1) has an almost periodic solution $x_{\varphi}=\left(x_{\varphi_{1}}, x_{\varphi_{2}}\right)^{T}$, where

$$
\begin{aligned}
x_{\varphi_{1}}(t)= & \int_{-\infty}^{t} e_{-\alpha}(t, \sigma(s))\left(\sum_{j=1}^{m} \beta_{j}(s) e^{-\gamma_{j}(s) \varphi_{1}\left(s-\tau_{j}(s)\right)}\right. \\
& \left.-c(s) \varphi_{2}(s-\theta(s)) \varphi_{1}(s)\right) \Delta s, \\
x_{\varphi_{2}}(t)= & \int_{-\infty}^{t} e_{-a}(t, \sigma(s))\left(b(s) \varphi_{1}(s-\eta(s))\right) \Delta s .
\end{aligned}
$$

Define a mapping $T: \mathbb{X}^{*} \rightarrow \mathbb{X}^{*}$, by $T \varphi(t)=x_{\varphi}(t), \forall \varphi \in \mathbb{X}^{*}$. We can observe that $\mathbb{X}^{*}$ is a closed subset of $\mathbb{X}$ obviously. For any $\varphi \in \mathbb{X}^{*}$, by use of $\left(H_{2}\right)$, we have

$$
\begin{aligned}
& x_{\varphi_{1}}(t) \leq \int_{-\infty}^{t} e_{-\alpha}(t, \sigma(s))\left(\sum_{j=1}^{m} \beta_{j}^{+}\right) \Delta s \leq \frac{\sum_{j=1}^{m} \beta_{j}^{+}}{\alpha^{-}}=M_{1}, \\
& x_{\varphi_{2}}(t) \leq \int_{-\infty}^{t} e_{-a}(t, \sigma(s))\left(b^{+} M_{1}\right) \Delta s \leq \frac{b^{+} M_{1}}{a^{-}}=M_{2} .
\end{aligned}
$$

We also have

$$
\begin{aligned}
x_{\varphi_{1}}(t) & \geq \int_{-\infty}^{t} e_{-\alpha}(t, \sigma(s))\left(\sum_{j=1}^{m} \beta_{j}^{-} e^{-\gamma_{j}^{+} M_{1}}-c^{+} M_{1} M_{2}\right) \Delta s \\
& \geq \frac{1}{\alpha^{+}}\left(\sum_{j=1}^{m} \beta_{j}^{-} e^{-\gamma_{j}^{+} M_{1}}-c^{+} M_{1} M_{2}\right)=m_{1}, \\
x_{\varphi_{2}}(t) & \geq \int_{-\infty}^{t} e_{-a}(t, \sigma(s))\left(b^{-} m_{1}\right) \Delta s \geq \frac{b^{-} m_{1}}{a^{+}}=m_{2} .
\end{aligned}
$$


Therefore, the mapping $T$ is a self-mapping from $\mathbb{X}^{*}$ to $\mathbb{X}^{*}$. Next, we prove that the mapping $T$ is a contraction mapping on $\mathbb{X}^{*}$. By the mean value theorem, we get

$$
\left|e^{-x}-e^{-y}\right|=\left|-e^{-\xi}\right| \cdot|x-y|, \quad x \leq \xi \leq y .
$$

For any $\varphi=\left(\varphi_{1}, \varphi_{2}\right)^{T}, \psi=\left(\psi_{1}, \psi_{2}\right)^{T} \in \mathbb{X}^{*}$, we obtain

$$
\begin{aligned}
& \left|(T \varphi)_{1}(t)-(T \psi)_{1}(t)\right| \\
& \leq \int_{-\infty}^{t} e_{-\alpha}(t, \sigma(s))\left(\sum_{j=1}^{m} \beta_{j}(s)\left(e^{-\gamma_{j}(s) \varphi_{1}\left(s-\tau_{j}(s)\right)}-e^{-\gamma_{j}(s) \psi_{1}\left(s-\tau_{j}(s)\right)}\right)\right. \\
& \left.\quad-c(s)\left(\varphi_{1}(s) \varphi_{2}(s-\theta(s))-\psi_{1}(s) \psi_{2}(s-\theta(s))\right)\right) \Delta s \\
& \leq \int_{-\infty}^{t} e_{-\alpha}(t, \sigma(s)) \sum_{j=1}^{m} \beta_{j}(s) \gamma_{j}(s)\left|\varphi_{1}\left(s-\tau_{j}(s)\right)-\psi_{1}\left(s-\tau_{j}(s)\right)\right| \\
& \quad+c(s)\left(\left|\varphi_{1}(s)\right| \cdot\left|\varphi_{2}(s-\theta(s))-\psi_{2}(s-\theta(s))\right|\right. \\
& \left.\quad+\left|\psi_{2}(s-\theta(s))\right| \cdot\left|\varphi_{1}(s)-\psi_{1}(s)\right|\right) \Delta s \\
& \leq \frac{1}{\alpha^{-}}\left(\sum_{j=1}^{m} \beta_{j}^{+} \gamma_{j}^{+}+c^{+}\left(M_{1}+M_{2}\right)\right) \cdot\|\varphi-\psi\|_{\mathbb{X}}, \\
& \left|(T \varphi)_{2}(t)-(T \psi)_{2}(t)\right| \\
& =\int_{-\infty}^{t} e_{-a}(t, \sigma(s)) b(s)\left(\varphi_{2}(s-\eta(s))-\psi_{2}(s-\eta(s))\right) \Delta s \\
& \leq \frac{1}{a^{-}} b^{+} \cdot\|\varphi-\psi\| \|_{\mathbb{X} .}
\end{aligned}
$$

It follows from $\left(H_{2}\right)$ that

$$
\|T \varphi-T \psi\|_{\mathbb{X}} \leq \max \left\{\frac{1}{\alpha^{-}}\left(\sum_{j=1}^{m} \beta_{j}^{+} \gamma_{j}^{+}+c^{+}\left(M_{1}+M_{2}\right)\right), \frac{b^{+}}{a^{-}}\right\}\|\varphi-\psi\|_{\mathbb{X}}<\|\varphi-\psi\|_{\mathbb{X}},
$$

which implies that $T$ is a contraction. By the fixed point theorem in Banach space, $T$ has a unique fixed point $\varphi^{*}=\left(\varphi_{1}^{*}, \varphi_{2}^{*}\right) \in \mathbb{X}^{*}$ such that $T \varphi^{*}=\varphi^{*}$. In view of (4.1), we receive that $\varphi^{*}$ is a solution of system (1.2). Therefore, (1.2) has a unique almost periodic positive solution in region $\mathbb{X}^{*}$. The proof is completed.

Theorem 4.2 Assume that $\left(H_{1}\right)$ and $\left(H_{2}\right)$ holds, the unique positive almost periodic solution in the region $\mathbb{X}^{*}$ is exponentially stable.

Proof By Theorem 4.1, system (1.2) has a positive almost periodic solution $w^{*}(t)=$ $\left(x^{*}(t), u^{*}(t)\right)^{T}$ in the region $\mathbb{X}^{*}$. Let $w(t)=(x(t), u(t))^{T}$ be any arbitrary solution of (1.2) with initial value $\varphi(t)=\left(\varphi_{1}(t), \varphi_{2}(t)\right)^{T}$ for $t \in[-\tau, 0)_{\mathbb{T}}$. Let $\varphi^{*}=\left(\varphi_{1}^{*}(t), \varphi_{2}^{*}(t)\right)^{T}$ be the initial function of $w^{*}(t), w^{*}\left(t, \varphi^{*}\right)=\varphi^{*}(t)$ for $t \in[-\tau, 0)_{\mathbb{T}}$. Now we prove $\left(x^{*}(t), u^{*}(t)\right)^{T}$ is exponential stable. 
Let $y_{1}(t)=x(t)-x^{*}(t), y_{2}(t)=u(t)-u^{*}(t)$, then we have

$$
\begin{aligned}
y_{1}^{\Delta}(t)= & \left(x(t)-x^{*}(t)\right)^{\Delta} \\
= & -\alpha(t)\left(x(t)-x^{*}(t)\right)+\sum_{j=1}^{m} \beta_{j}(t)\left\{e^{-\gamma_{j}(t) x\left(t-\tau_{j}(t)\right)}-e^{-\gamma_{j}(t) x^{*}\left(t-\tau_{j}(t)\right)}\right\} \\
& -c(t)\left\{u(t-\theta(t)) x(t)-u^{*}(t-\theta(t)) x^{*}(t)\right\}, \quad j=1,2, \ldots, m, \\
y_{2}^{\Delta}(t)= & \left(u(t)-u^{*}(t)\right)^{\Delta} \\
= & -a(t)\left(u(t)-u^{*}(t)\right)+b(t)\left(x(t-\eta(t))-x^{*}(t-\eta(t))\right) .
\end{aligned}
$$

Let $h(t)=\sum_{j=1}^{m} \beta_{j}(t)\left\{e^{-\gamma_{j}(t) x\left(t-\tau_{j}(t)\right)}-e^{-\gamma_{j}(t) x^{*}\left(t-\tau_{j}(t)\right)}\right\}-c(t)\left\{u(t-\theta(t)) x(t)-u^{*}(t-\theta(t)) x^{*}(t)\right\}, j=$ $1,2, \ldots, m$, then is follows from (4.2) that

$$
\begin{aligned}
& y_{1}^{\Delta}(t)=-\alpha(t) y_{1}(t)+h(t), \\
& y_{2}^{\Delta}(t)=-a(t) y_{2}(t)+b(t) y_{1}(t-\eta(t)) .
\end{aligned}
$$

Thus, we see that $y_{1}(t), y_{2}(t)$ can be expressed as follows:

$$
\begin{aligned}
& y_{1}(t)=y_{1}\left(t_{0}\right) e_{-\alpha}\left(t, t_{0}\right)+\int_{t_{0}}^{t} e_{-\alpha}(t, \sigma(s)) h(s) \Delta s, \\
& y_{2}(t)=y_{2}\left(t_{0}\right) e_{-a}\left(t, t_{0}\right)+\int_{t_{0}}^{t} e_{-a}(t, \sigma(s)) b(s) y_{1}(s-\eta(s)) \Delta s, \quad t \geq t_{0}, t_{0} \in[-\tau, 0)_{\mathbb{T}},
\end{aligned}
$$

which implies that

$$
\begin{aligned}
& y_{1}(t)=e_{-\alpha}\left(t, t_{0}\right)\left(\varphi_{1}\left(t_{0}\right)-\varphi_{1}^{*}\left(t_{0}\right)\right)+\int_{t_{0}}^{t} e_{-\alpha}(t, \sigma(s)) h(s) \Delta s, \\
& y_{2}(t)=e_{-a}\left(t, t_{0}\right)\left(\varphi_{2}\left(t_{0}\right)-\varphi_{2}^{*}\left(t_{0}\right)\right)+\int_{t_{0}}^{t} e_{-a}(t, \sigma(s)) b(s) y_{1}(s-\eta(s)) \Delta s .
\end{aligned}
$$

Note that

$$
\begin{aligned}
|h(t)| \leq & \sum_{j=1}^{m}\left|\beta_{j}(t)\right| \cdot\left|\left\{e^{-\gamma_{j}(t) x\left(t-\tau_{j}(t)\right)}-e^{-\gamma_{j}(t) x^{*}\left(t-\tau_{j}(t)\right)}\right\}\right| \\
& +|c(t)| \cdot\left\{\left|u(t-\theta(t)) x(t)-u^{*}(t-\theta(t)) x(t)\right|\right. \\
& \left.+\left|u^{*}(t-\theta(t)) x(t)-u^{*}(t-\theta(t)) x^{*}(t)\right|\right\} .
\end{aligned}
$$

By the mean value theorem, we obtain

$$
\begin{aligned}
& \left|e^{-\gamma_{j}(t) x\left(t-\tau_{j}(t)\right)}-e^{-\gamma_{j}(t) x^{*}\left(t-\tau_{j}(t)\right)}\right| \\
& \quad=\left|-e^{-\xi_{j}} \gamma_{j}(t)\left[x\left(t-\tau_{j}(t)\right)-x^{*}\left(t-\tau_{j}(t)\right)\right]\right| \\
& \quad \leq e^{-\xi_{j}}\left|\gamma_{j}(t)\right| \cdot\left|x\left(t-\tau_{j}(t)\right)-x^{*}\left(t-\tau_{j}(t)\right)\right| \\
& \quad \leq \gamma_{j}^{+}\left|y_{1}\left(t-\tau_{j}(t)\right)\right| .
\end{aligned}
$$


Then we get

$$
|h(t)| \leq \sum_{j=1}^{m} \beta_{j}^{+} \gamma_{j}^{+}\left|y_{1}\left(t-\tau_{j}(t)\right)\right|+c^{+}\left(M_{1}\left|y_{2}(t-\theta(t))\right|+M_{2}\left|y_{1}(t)\right|\right) .
$$

Hence, by (4.5) and (4.6), we obtain

$$
\begin{aligned}
\left|y_{1}(t)\right| \leq & e_{-\alpha}\left(t, t_{0}\right)\left|\varphi_{1}\left(t_{0}\right)-\varphi_{1}^{*}\left(t_{0}\right)\right| \\
& +\int_{t_{0}}^{t} e_{-\alpha}(t, \sigma(s))\left\{\sum_{j=1}^{m} \beta_{j}^{+} \gamma_{j}^{+}\left|y_{1}\left(s-\tau_{j}(s)\right)\right|\right. \\
& \left.+c^{+}\left(M_{1}\left|y_{2}(s-\theta(s))\right|+M_{2}\left|y_{1}(s)\right|\right)\right\} \Delta s, \\
& \\
\left|y_{2}(t)\right| \leq & e_{-a}\left(t, t_{0}\right)\left|\varphi_{2}\left(t_{0}\right)-\varphi_{2}^{*}\left(t_{0}\right)\right|+\int_{t_{0}}^{t} e_{-a}(t, \sigma(s)) b^{+}\left|y_{1}(s-\eta(s))\right| \Delta s .
\end{aligned}
$$

Taking the norm at both sides of (4.7), we have

$$
\begin{aligned}
\|y(t)\| \leq & e_{-\alpha}\left(t, t_{0}\right)\left\|\varphi-\varphi^{*}\right\| \\
& \quad+\int_{t_{0}}^{t} e_{-\alpha}(t, \sigma(s))\left\{\sum_{j=1}^{m} \mid \beta_{j}^{+} \gamma_{j}^{+}+c^{+}\left(M_{1}+M_{2}\right)\right\}\|y\| \Delta s, \\
\|y(t)\| \leq & e_{-a}\left(t, t_{0}\right)\left\|\varphi-\varphi^{*}\right\|+\int_{t_{0}}^{t} e_{-a}(t, \sigma(s)) b^{+}\|y\| \Delta s .
\end{aligned}
$$

Then we get

$$
\begin{aligned}
\frac{\|y(t)\|}{e_{-\alpha}\left(t, t_{0}\right)} \leq\left\|\varphi-\varphi^{*}\right\|+\int_{t_{0}}^{t} \frac{\|y\|}{e_{-\alpha}\left(\sigma(s), t_{0}\right)} \rho \Delta s, \\
\frac{\|y(t)\|}{e_{-a}\left(t, t_{0}\right)} \leq\left\|\varphi-\varphi^{*}\right\|+\int_{t_{0}}^{t} \frac{\|y\|}{e_{-a}\left(\sigma(s), t_{0}\right)} b^{+} \Delta s,
\end{aligned}
$$

where $\rho=\sum_{j=1}^{m} \beta_{j}^{+} \gamma_{j}^{+}+c^{+}\left(M_{1}+M_{2}\right)$.

By the Gronwall inequality [30], we have

$$
\begin{aligned}
\|y(t)\| & \leq\left\|\varphi-\varphi^{*}\right\| e_{\rho}\left(t, t_{0}\right) e_{-\alpha}\left(t, t_{0}\right) \\
& \leq\left\|\varphi-\varphi^{*}\right\| e_{-\left(\alpha^{-}-\rho\right)}\left(t, t_{0}\right), \\
\|y(t)\| & \leq\left\|\varphi-\varphi^{*}\right\| e_{b^{+}}\left(t, t_{0}\right) e_{-a}\left(t, t_{0}\right) \\
& \leq\left\|\varphi-\varphi^{*}\right\| e_{-\left(a^{-}-b^{+}\right)}\left(t, t_{0}\right) .
\end{aligned}
$$

From the condition $\left(\mathrm{H}_{2}\right)$, we get

$$
\|y(t)\|=\left\|w(t)-w^{*}(t)\right\| \leq\left\|\varphi-\varphi^{*}\right\| e_{-\lambda}\left(t, t_{0}\right),
$$

where $\lambda=\min \left\{\alpha^{-}-\rho, a^{-}-b^{+}\right\}>0$. Hence, (4.11) implies that $w^{*}(t)$ is exponentially stable. The proof of Theorem 4.2 is completed. 
Remark 4.1 If $\mathbb{T}=\mathbb{R}$ and $\mathbb{T}=\mathbb{Z}$, then (1.2) reduces to

$$
\begin{aligned}
x^{\prime}(t)= & -\alpha(t) x(t)+\sum_{j=1}^{m} \beta_{j}(t) e^{-\gamma_{j}(t) x\left(t-\tau_{j}(t)\right)} \\
& -c(t) u(t-\theta(t)) x(t), \quad j=1,2, \ldots, m, \\
u^{\prime}(t)= & -a(t) u(t)+b(t) x(t-\eta(t)), \quad t \in \mathbb{R},
\end{aligned}
$$

and

$$
\begin{aligned}
x(k+1)-x(k)= & -\alpha(k) x(k)+\sum_{j=1}^{m} \beta_{j}(k) e^{-\gamma_{j}(k) x\left(k-\tau_{j}(k)\right)} \\
& -c(k) u(k-\theta(k)) x(k), \quad j=1,2, \ldots, m, \\
u(k+1)-u(k)= & -a(k) u(k)+b(k) x(k-\eta(k)), \quad k \in \mathbb{Z},
\end{aligned}
$$

respectively.

\section{Numerical simulation}

Example 1 Consider the following system on time scales:

$$
\begin{aligned}
x^{\Delta}(t)= & -(0.8+0.2 \cos (\sqrt{2} t)) x(t) \\
& +\sum_{j=1}^{3}(0.21+0.01|\sin (\sqrt{3} t)|) e^{-(0.21+0.01|\cos t|) x(t-2)} \\
& -(0.1+0.1|\cos t|) u(t-3) x(t), \\
u^{\Delta}(t)= & -(0.25+0.1 \cos t) u(t)+(0.1+0.01 \sin (\sqrt{2} t)) x(t-2) .
\end{aligned}
$$

By direct calculation, we can get

$$
\begin{array}{lll}
\alpha^{+}=1, & \alpha^{-}=0.6, \quad \beta_{j}^{+}=0.22, & \beta_{j}^{-}=0.2, \\
\gamma_{j}^{+}=0.22, & \gamma_{j}^{-}=0.2 & (j=1,2,3) \\
c^{+}=0.2, & c^{+}=0.1, \quad a^{+}=0.25, & a^{-}=0.15, \quad c^{+}=0.2, \quad c^{-}=0.1,
\end{array}
$$

then

$$
\begin{aligned}
& M_{1}=\frac{\sum_{j=1}^{3} \beta_{j}^{+}}{\alpha^{-}}=1.1, \quad M_{2}=\frac{b^{+} M_{1}}{a^{-}}=0.0202, \\
& \sum_{j=1}^{3} \beta_{j}^{-} e^{-\gamma_{j}^{+} M_{1}}=0.4710>c^{+} M_{1} M_{2}=0.2240, \\
& \sum_{j=1}^{3} \beta_{j}^{+} \gamma_{j}^{+}+c^{+}\left(M_{1}+M_{2}\right)=0.3692<\alpha^{-}=0.6, \quad b^{+}=0.11<a^{-}=0.15,
\end{aligned}
$$


Figure $1 \mathbb{T}=\mathbb{R}$, numeric simulations of the continuous situation of system (5.1), the initial conditions $(x(0), u(0))=(0.8,0.5),(0.2,0.3)$ and $(0.3,0.4)$, respectively

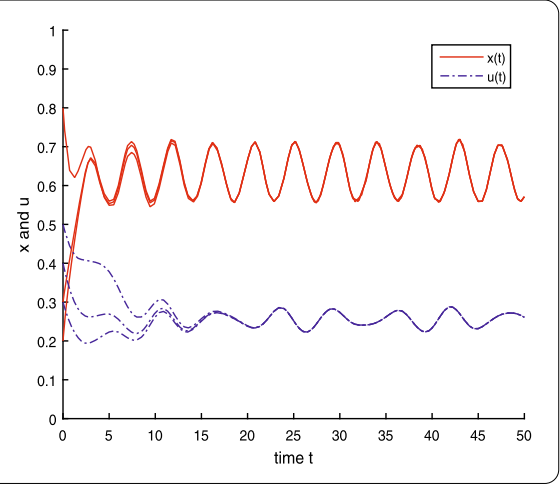

Figure $2 \mathbb{T}=\mathbb{Z}$, numeric simulations of discrete situation of system (5.1), the initial conditions $(x(0), u(0))=(0.2,0.3)$, $(1,0.5)$ and $(0.8,0.6)$, respectively

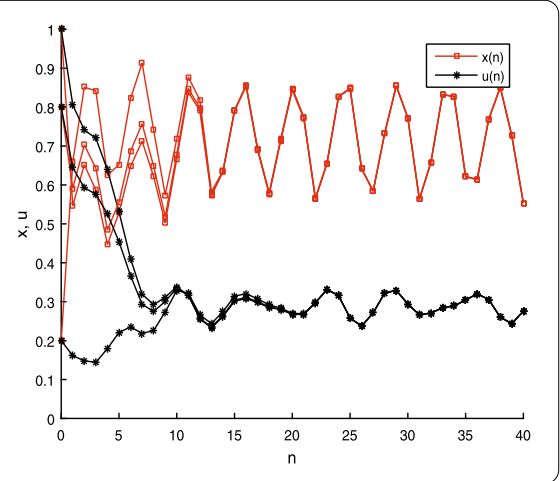

that is, conditions $\left(H_{1}\right)$ and $\left(H_{2}\right)$ hold. According to Theorem 4.1 and Theorem 4.2, system (5.1) has a unique positive almost periodic solution, which is exponentially stable. For dynamic simulations of system (5.1) with $\mathbb{T}=\mathbb{R}$ and $\mathbb{T}=\mathbb{Z}$, see Figs. 1 and 2 , respectively.

\section{Discussion}

Comparing with the previous relative results of [28], our investigation confirms that the feedback control does not have any influence on the permanence of the system. Our results improve and complement some known results to some degree in the literature. The explorations in this paper reveal that, when we deal with almost periodic solution for differential equations and difference equations, it is unnecessary to prove results for continuous and discrete cases separately. Our results could unify such problems in the framework of dynamic equations on time scales.

Funding

This work is supported by the Natural Science Foundation of Fujian Province (2019J01651).

\section{Competing interests}

The authors declare that there is no conflict of interests.

\section{Authors' contributions}

All authors contributed equally to the writing of this paper. All authors read and approved the final manuscript.

\section{Author details}

'Department of Computer Engineering, Fuzhou University Zhicheng College, Fuzhou, P.R. China. ${ }^{2}$ Department of Mathematics, Minjiang University, Fuzhou, P.R. China. 


\section{Publisher's Note}

Springer Nature remains neutral with regard to jurisdictional claims in published maps and institutional affiliations.

\section{Received: 30 October 2019 Accepted: 24 December 2019 Published online: 08 January 2020}

\section{References}

1. Hilger, S.: Analysis on measure chains - a unified approach to continuous and discrete calculus. Results Math. 18, 18-56 (1990)

2. Yao, Z.J.: Existence and exponential stability of unique almost periodic solution for Lasota-Wazewska red blood cell model with perturbation on time scales. Math. Methods Appl. Sci. 40(13), 1083-1089 (2017)

3. Yang, L., Li, Y.K., Wu, W.Q.: $C^{n}$-Almost periodic functions and an application to a Lasota-Wazewska model on time scales. J. Appl. Math. 2014, Article ID 321328 (2014)

4. Wang, C., Agarwal, R.P.: Almost periodic solution for a new type of neutral impulsive stochastic Lasota-Wazewska timescale model. Appl. Math. Lett. 70, 58-65 (2017)

5. Li, Y.K., Li, B.: Existence and exponential stability of positive almost periodic solution for Nicholson's blowflies models on time scales. SpringerPlus 5, 1096 (2016)

6. Wang, Q.L., Liu, Z.J.: Existence and stability of positive almost periodic solutions for a competitive system on time scales. Math. Comput. Simul. 138, 65-77 (2017)

7. Fan, Y.H., Yu, Y.Y., Wang, L.L.: Some differential inequalities on time scales and their applications to feedback control systems. Discrete Dyn. Nat. Soc. 2017, Article ID 9195613 (2017)

8. Wang, C.: Existence and exponential stability of piecewise mean-square almost periodic solutions for impulsive stochastic Nicholson's blowflies model on time scales. Appl. Math. Comput. 248, 101-112 (2014)

9. Wang, L.L., Fan, Y.H.: Permanence and existence of periodic solutions for a Nicholson's blowflies model with feedback control and delay on time scales. Discrete Dyn. Nat. Soc. 2018, Article ID 3403127 (2018)

10. Li, Y.K., Yang, L.: Almost periodic solutions for neutral-type BAM neural networks with delays on time scales. J. Appl. Math. 2013, Article ID 942309 (2013)

11. Gopalsamy, K., Weng, P.X.: Feedback regulation of logistic growth. Int. J. Math. Sci. 16(1), 177-192 (1993)

12. Chen, F.D.: Permanence of a discrete $\mathrm{N}$-species cooperation system with time delays and feedback controls. Appl. Math. Comput. 186(1), 23-29 (2007)

13. Li, Y.K., Zhang, T.W.: Global asymptotical stability of a unique almost periodic solution for enterprise clusters based on ecology theory with time-varying delays and feedback controls. Commun. Nonlinear Sci. Numer. Simul. 17, 904-913 (2012)

14. Chen, F.D., Yang, J.H., Chen, L.J.: On a mutualism model with feedback controls. Appl. Math. Comput. 214(2), 581-587 (2009)

15. Chen, F.D., Yang, J.H., Chen, L.J.: Note on the persistent property of a feedback control system with delays. Nonlinear Anal., Real World Appl. 11, 1061-1066 (2010)

16. Chen, L.J., Xie, X.D.: Permanence of a $n$-species cooperation system with continuous time delays and feedback controls. Nonlinear Anal., Real World Appl. 12(1), 34-38 (2011)

17. Chen, X.Y., Shi, C.L., Wang, Y.Q.: Almost periodic solution of a discrete Nicholson's blowflies model with delay and feedback control. Adv. Differ. Equ. 2016(1), 185 (2016)

18. Li, Z., Han, M.A., Chen, F.D.: Influence of feedback controls on an autonomous Lotka-Volterra competitive system with infinite delays. Nonlinear Anal., Real World Appl. 14, 402-413 (2013)

19. Chen, X.Y., Shi, C.L.: Permanence of a Nicholson's blowflies model with feedback control and multiple time-varying delays. Chin. Q. J. Math. 30(1), 153-158 (2015)

20. Chen, X.Y: Almost periodic solution of a delayed Nicholson's blowflies model with feedback control. Commun. Math. Biol. Neurosci. 2015, Article ID 10 (2015)

21. Chen, X.Y., Shi, C.L.: Permanence and global attractivity of a discrete Nicholson's blowflies model with delay. J. Math. Res. Appl. 37(2), 233-241 (2017)

22. Shi, L., Liu, H., Wei, Y.M., et al.: The permanence and periodic solution of a competitive system with infinite delay, feedback control, and Allee effect. Adv. Differ. Equ. 2018, 400 (2018)

23. Wazewska-Czyzewska, M., Lasota, A.: Mathematical problems of the dynamics of red blood cells system. Ann. Pol. Math. Soc. 6, 23-40 (1976)

24. Shao, J.Y: Pseudo almost periodic solutions for a Lasota-Wazewska model with an oscillating death rate. Appl. Math. Lett. 43, 90-95 (2015)

25. Zhou, H., Jiang, W.: Existence and stability of positive almost periodic solution for stochastic Lasota-Wazewska model. J. Appl. Math. Comput. 47, 61-71 (2015)

26. Xiao, S.L.: Delay effect in the Lasota-Wazewska model with multiple time-varying delays. Int. J. Biomath. 11(1), 1850013 (11 pages) (2018)

27. Stamov, G.T:: On the existence of almost periodic solutions for the impulsive Lasota-Wazewska model. Appl. Math. Lett. 22, 516-520 (2009)

28. Huang, Z.D., Gong, S.H., Wang, L.J.: Positive almost periodic solution for a class of Lasota-Wazewska model with multiple time-varying delays. Comput. Math. Appl. 6, 755-760 (2011)

29. Zhao, L., Qin, B., Chen, F.D.: Permanence and global stability of a may cooperative system with strong and weak cooperative partners. Adv. Differ. Equ. 2018, 172 (2018)

30. Bohner, M., Peterson, A.: Dynamic Equations on Time Scales: An Introduction with Applications. Birkhauser, Boston (2001)

31. Hu, M., Wang, L.L.: Dynamic inequalities on time scales with applications in permanence of predator-prey system. Discrete Dyn. Nat. Soc. 2012, Article ID 281052 (2012)

32. Li, Y.K., Wang, C.: Almost periodic functions on time scales and applications. Discrete Dyn. Nat. Soc. 2011, Article ID $727068(2011)$

33. Zhang, J.M., Fan, M., Zhu, H.P.: Existence and roughness of exponential dichotomies of linear dynamic equations on time scales. Comput. Math. Appl. 59, 2658-2675 (2010) 